\title{
Atypical presentation of melorheostosis with soft tissues involvement: a case report
}

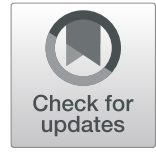

Kok King Chia ${ }^{* *} \mathbb{D}$, Juhara Haron ${ }^{1}$ and Nik Fatimah Salwati Nik Malek ${ }^{2}$

\begin{abstract}
Background: Melorheostosis is a skeletal disorder giving rise to a dripping wax appearance. The exact cause is still unclear, and the diagnosis is always challenging due to its wide differential diagnoses. Soft tissue involvement of melorheostosis has been reported in previous literatures but it is a rare phenomenon.

Case presentation: A 10-year-old child with melorheostosis presented with hematuria. Ultrasonography (USG) identified a cystic lesion in the right hemipelvis adjacent to the urinary bladder which was initially regarded as an ovarian cyst. Computed tomography (CT) confirmed the cystic lesion as a fusiform right internal iliac artery aneurysm, as well as multiple right retroperitoneal and right lower limb capillary hemangiomas with uterine involvement. Hence, the final diagnosis was atypical melorheostosis with vascular malformations. The patient was managed conservatively with Sirolimus therapy for the vascular anomalies.
\end{abstract}

Conclusion: Albeit the rare involvement of soft tissues, careful search for vascular malformation is recommended in melorheostosis.

Keywords: Melorheostosis, Osteosclerosis, Skeletal dysplasia, Capillary hemangioma, Iliac artery aneurysm

\section{Background}

Melorheostosis (Leri's disease) is a rare benign nonhereditary mesenchymal sclerotic bone dysplasia, characterized by regions of dripping wax or flowing candle wax appearance due to cortical hyperostosis. The name was

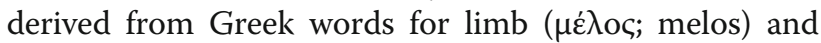
flow ( $\dot{\rho} \varepsilon$; r réō), for its characteristic appearance of flowing hyperostosis [1].

The prevalence is about 0.9 cases per million population and there are approximately 400 cases described in the literature [2]. The estimated incidence of melorheostosis is 1 in 1,000,000. Melorheostosis affects men and women in equal proportions with occurrence reported in child and adult populations [3].

\footnotetext{
* Correspondence: drkkchia@gmail.com

'Department of Radiology, School of Medical Sciences, Universiti Sains

Malaysia, Kelantan, Malaysia

Full list of author information is available at the end of the article
}

Long bones are commonly affected, although it can affect any segment of the bones. It tends to be segmental and unilateral [4]. Soft tissue involvement is extremely rare with few reported literatures. Treatment is primarily conservative for pain relief. However, the treatment of soft tissue involvement is variable depending on the types of soft tissues component $[5,6]$.

\section{Case presentation}

A 10-year-old girl with an underlying skeletal dysplasia presented with acute hematuria. She had right thigh to calf area swelling with intermittent right lower limb pain and café-au-lait spots since the age of three. She was initially diagnosed with osteogenesis imperfecta until she presented to our treatment center with episodic vaginal bleeding when she was 6 years old, which was thought to be precocious

\section{Springer Open}


puberty. Hence, a diagnosis of McCune-Albright syndrome was made due to precocious puberty, café au lait spots, and skeletal dysplasia. A transabdominal USG was performed by the gynecologist who revealed a right ovarian cyst.

On physical examination, there was a right knee fixed deformity with swelling of right thigh and calf, as well as patchy cutaneous dark spots. Per speculum examination was unremarkable. Her laboratory results for thyroid function, erythrocyte sedimentation rate (ESR), rheumatoid factor, luteinizing hormone ( $\mathrm{LH})$, follicle-stimulating hormone (FSH), estradiol, serum complements, serum cortisol, serum calcium, serum lactate dehydrogenase (LDH), growth hormone, serum prolactin, and antistreptolysin-O titer (ASOT) are within normal limits. Plain radiographs showed multiple sclerotic cortical hyperostosis with dripping candle wax appearance over the right ilium, T12 vertebra with hypoplastic right 12 th rib, right ribs, right femur, right tibia and fibula, right 2nd-5th metatarsal, and right cuboid (Figs. 1 and 2), right scapula, right humerus, right radius, and right hand. Transabdominal USG showed a small right ovarian cyst and thickened urinary bladder wall with blood clots in the urinary bladder. She was referred to the pediatric surgical team for cystitis and to exclude rhabdomyosarcoma.

A repeated transabdominal USG by a consultant radiologist revealed a right internal iliac artery aneurysm, which was mistaken as an ovarian cyst (Fig. 3). USG of the right thigh showed thickened echogenic subcutaneous fat with low-flow vascular malformation extending from the right inguinal region till the lower leg. CT angiography of lower limbs confirmed the right internal iliac artery fusiform aneurysm with vascular malformations extending from retroperitoneum to right lower limb with uterine involvement, likely capillary hemangiomas (Figs. 4 and 5). There was no evidence of aneurysm-ureteric fistula. Hence, the final diagnosis was consistent with atypical melorheostosis with vascular malformations. She was treated conservatively with Sirolimus for the vascular malformations.

\section{Discussion}

Melorheostosis or Leri's disease was first described in 1922 by Leri and Joanny [7]. It was previously hypothesized that melorheostosis was caused by somatic mutations in LEMD3 that is only present in the affected tissues [8]. However, recent discovery in molecular biology had identified the causes of melorheostosis. Kang et al. described somatic mosaicism for MAP2K1-activating mutations and SMAD3

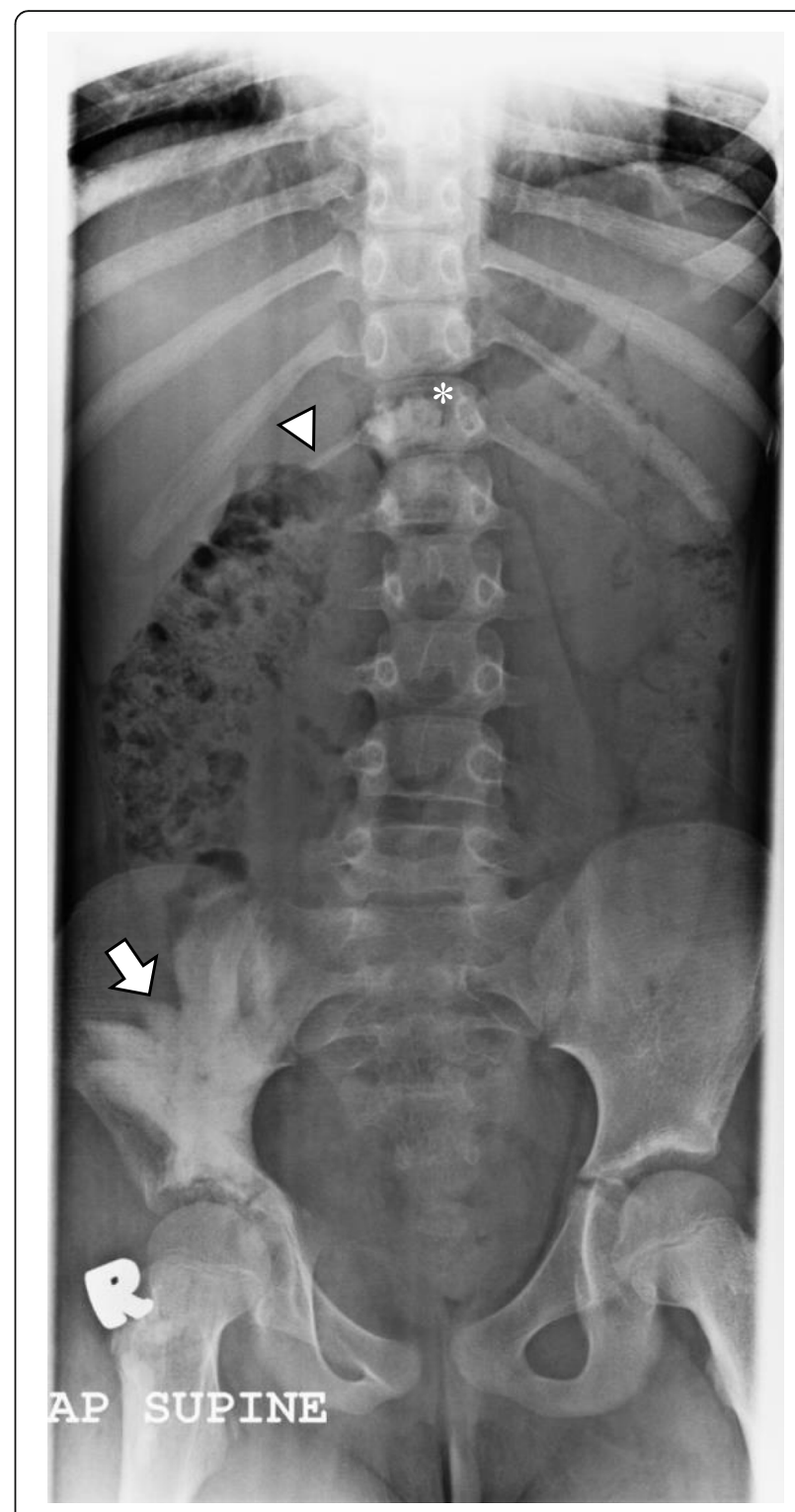

Fig. 1 Flowing candle wax appearance at the right ilium (big arrow) and T12 vertebra (asterisk) with hypoplastic right 12th rib (arrowhead) on plain radiograph

mutations as the causes for the classical "dripping candle wax" appearance in melorheostosis $[9,10]$.

A wide spectrum of soft tissue involvement in melorheostosis was reported in previous literatures, including subcutaneous fibrosis, erythema, linear scleroderma-like patches, ectopic bone formation, hypertrichosis, fibromas, fibrolipomas, and vascular malformations (e.g., capillary hemangiomas, lymphangiectasia, or arterial aneurysms). Soft tissue fibrosis with ligament and tendon retraction had been reported, manifesting as equinovarus or valgus or varus 


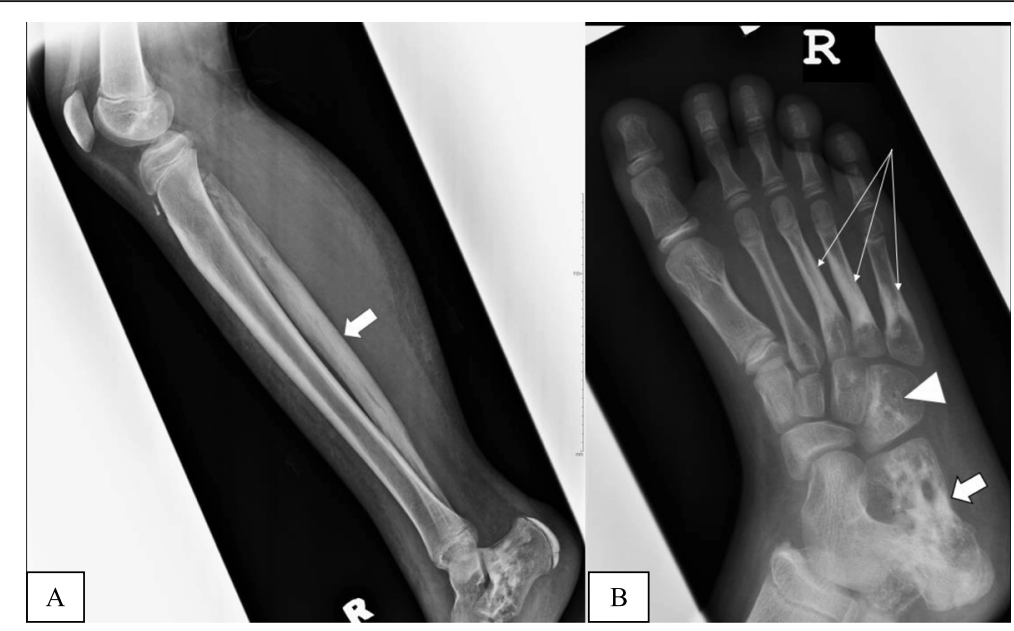

Fig. 2 Lateral plain radiograph of right tibia and fibula (a) and dorsal oblique of right foot (b) show sclerotic cortical changes of tibia and calcaneum (big arrows), cuboid (arrowhead), and 3rd-5th metatarsal bones (small arrows)

foot deformities. Early presentation and multiple limb involvement may predict a poorer prognosis in terms of complications [11].

Patients with melorheostosis may remain asymptomatic until late adolescence or early adulthood, though changes usually manifest in childhood. Main presenting symptom is often pain worsened by movements of the affected limb [1]. The onset of symptoms in our case started in early childhood with an atypical presentation of episodic vaginal bleeding. Distribution of bone lesions can be monomelic or hemimelic, with the former being the most common observation. It can also be monostotic or polyostotic, and occasionally bilateral involvement [12]. In polyostotic involvement, bone lesions typically cross joint spaces with a sclerotomal distribution. Whyte reported that limb length discrepancy may develop due to asymmetric early epiphyseal fusion [13]. We did not observe limb length discrepancy in our patient. Our patient experienced intermittent bone pain, predominantly in the right thigh. The skeletal lesions were distributed in a hemimelicpolyostotic fashion.

The soft tissue involvement in our case was vascular malformations and joint contracture of the right knee due to fibrosis. The right internal iliac artery fusiform aneurysm and capillary hemangiomas extending from retroperitoneum to the right lower limb with uterine involvement, explaining the occurrence of episodic vaginal bleeding. The capillary hemangiomas were mistaken for café-au-lait spots, which lead to the initial diagnosis of McCune-Albright syndrome.

Melorheostosis is classically diagnosed using plain radiograph with the pathognomonic appearance of "dripping candle wax or flowing candle wax" appearance due to cortical hyperostosis. CT and MRI may help to identify and delineate the extent of soft tissue involvement. Bone scan may show increased

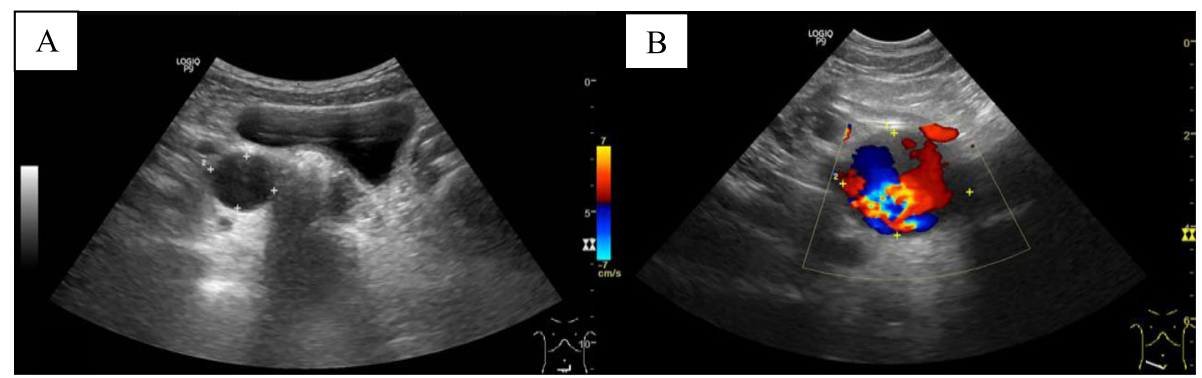

Fig. 3 Ultrasound of the pelvis shows cystic lesion in right hemipelvis (a). Turbulent flow on color Doppler within the cystic lesion (b) 


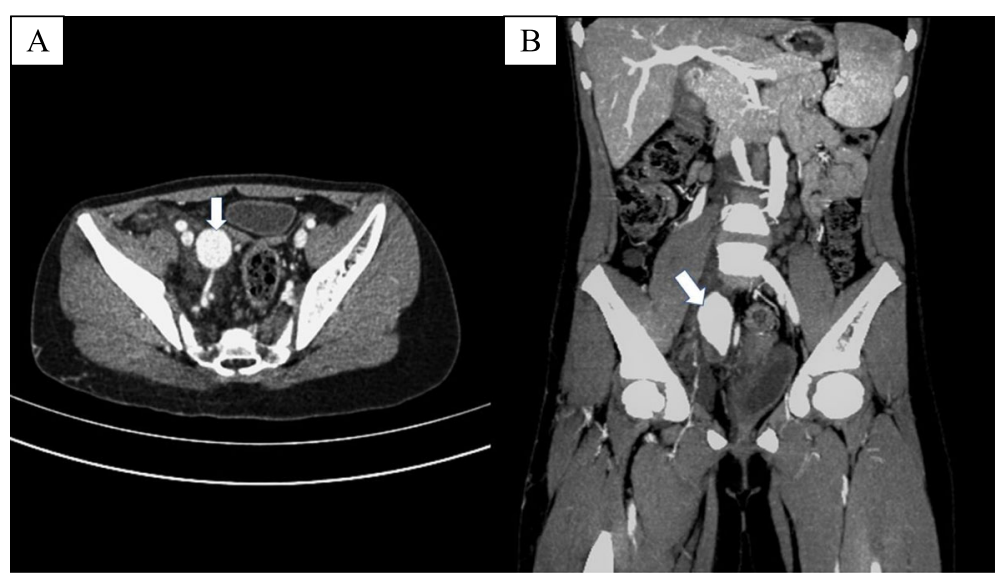

Fig. 4 Axial (a) and coronal (b) enhanced CT of abdomen and pelvis in the arterial phase demonstrate a fusiform aneurysm (arrow) of the proximal right internal iliac artery

radiotracer uptake in the osseous lesions, albeit plain radiograph is sufficient for the diagnosis of osseous involvement. Serum calcium, serum phosphorus, and alkaline phosphatase are often normal $[11,14]$. In our case, the blood results were within normal limit. Histopathologically, findings are nonspecific and often show a mixture of mature and immature bone in a dense formation with increased trabecular bone [3].

Treatment is conservative for pain alleviation and physiotherapy although in severe cases, surgical intervention may be required, including tendon release, osteotomies, and even amputation. A recent study found that melorheostosis can be treated with intravenous zoledronic acid and that treatment can be monitored by using a specific bone resorption marker
[15]. Sirolimus can be used to treat vascular malformation with proven safety and efficacy [6].

The differential diagnoses include osteopoikilosis, Buschke-Ollendorff syndrome, osteopathia striata, McCune-Albright syndrome, infantile cortical hyperostosis (Caffey disease), and desmoid tumors [16].

\section{Conclusion}

Albeit the rare involvement of soft tissue components, careful search for vascular malformation is recommended in melorheostosis. Early identification of soft tissue involvement in melorheostosis may prevent disastrous complications. Sirolimus is proven safe and efficacious in the treatment of vascular anomalies. However, interventional radiology may offer help in the case of complicated vascular malformation, resistant to pharmacotherapy.

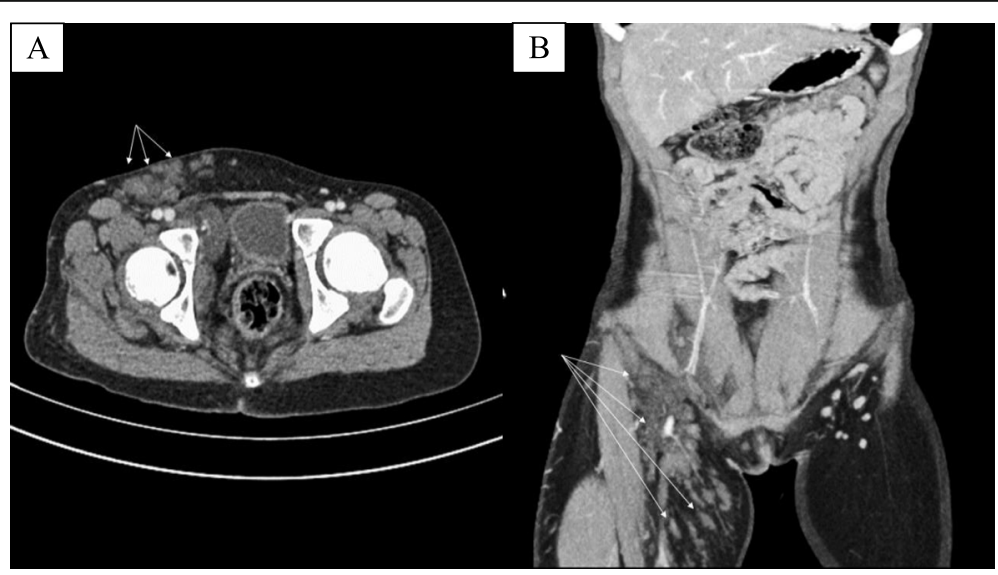

Fig. 5 Axial (a) and coronal (b) enhanced CT of abdomen and pelvis in the arterial phase demonstrate multiple enhancing subcutaneous lesions (small arrows) extending from retroperitoneum to visualized upper thigh suggestive of capillary hemangiomas 


\section{Abbreviations}

USG: Ultrasonography; CT: Computed tomography; MRI: Magnetic resonance imaging; ESR: Erythrocyte sedimentation rate; LH: Luteinizing hormone; FSH: Follicle-stimulating hormone; LDH: Lactate dehydrogenase; ASOT: Antistreptolysin-O titer

\section{Acknowledgements}

NIL.

\section{Authors' contributions}

Dr. CKK (1) had made substantial contributions to concept, design, acquisition, interpretation of data, and drafting the article. Dr. NFSNM (3) had made substantial contributions to intellectual content. Dr. JH (2) gave final approval of the version to be submitted. The authors have read and approved the transcript.

\section{Authors' information}

Medical Officer and Radiology Trainee, Department of Radiology, Hospital Universiti Sains Malaysia, 16150 Kota Bharu, Kelantan, Malaysia Chia Kok King (CKK)

Associate Professor and Consultant Radiologist, Department of Radiology, Hospital Universiti Sains Malaysia, 16150 Kota Bharu, Kelantan, Malaysia Juhara Haron $(\mathrm{JH})$

Head of Department and Consultant Radiologist, Department of Radiology, Hospital Sultanah Bahiyah, Km 6, Jalan Langgar, 05460 Alor Setar, Kedah,

Malaysia

Nik Fatimah Salwati Nik Malek (NFSNM)

\section{Funding}

NIL.

\section{Availability of data and materials}

All data generated or analyzed during this study are included in this published article.

\section{Ethics approval and consent to participate}

Not applicable.

\section{Consent for publication}

Written consent had been obtained from the parent of study participant.

\section{Competing interests}

The authors declare that they have no competing interests.

\section{Author details}

'Department of Radiology, School of Medical Sciences, Universiti Sains Malaysia, Kelantan, Malaysia. ${ }^{2}$ Department of Radiology, Hospital Sultanah Bahiyah, Alor Setar, Kedah, Malaysia.

Received: 18 November 2020 Accepted: 7 January 2021

Published online: 20 January 2021

\section{References}

1. Bansal A (2008) The dripping candle wax sign. Radiology 246:638-640. https://doi.org/10.1148/radiol.2462050537

2. Clifford PD, Jose J (2009) Melorheostosis. Am J Orthop (Belle Mead NJ) 38: 360-361

3. Alpoim BP, Rodrigues MEGR, Félix AJM et al (2013) Melorheostosis: a case report. Rev Bras Ortop 48:282-285

4. Long HT, Li KH, Zhu Y (2009) Case report: severe melorheostosis involving the ipsilateral extremities. Clin Orthop Relat Res 467:2738-2743. https://doi. org/10.1007/s11999-009-0890-y

5. Hasegawa S, Kanda S, Imada H et al (2017) Melorheostosis with recurrent soft-tissue components: a histologically confirmed case. Skeletal Radiol 46: 399-404. https://doi.org/10.1007/s00256-016-2562-9

6. Adams DM, Trenor CC, Hammill AM et al (2016) Efficacy and safety of sirolimus in the treatment of complicated vascular anomalies. Pediatrics 137 https://doi.org/10.1542/peds.2015-3257

7. Leri A (1922) Une affection non decrite des os. Hyperostose "en coulee" sur toute la longueur d'un membre ou "melorheostose". Bull Mem Soc Med Hop Paris 46:1141-1145
8. Hellemans J, Preobrazhenska O, Willaert A et al (2004) Loss-of-function mutations in LEMD3 result in osteopoikilosis, Buschke-Ollendorff syndrome and melorheostosis. Nat Genet 36:1213-1218. https://doi.org/10.1038/ ng1453

9. Kang $\mathrm{H}$, Jha S, Deng $\mathrm{Z}$ et al (2018) Somatic activating mutations in MAP2K1 cause melorheostosis. Nat Commun 9:1390. https://doi.org/10.1038/s41467018-03720-z

10. Kang $H$, Jha S, Ivovic A et al (2020) Somatic SMAD3-activating mutations cause melorheostosis by up-regulating the TGF- $\beta / S M A D$ pathway. J Exp Med 217. https://doi.org/10.1084/jem.20191499

11. Kotwal A, Clarke BL (2017) Melorheostosis: a rare sclerosing bone dysplasia. Curr Osteoporos Rep 15:335-342. https://doi.org/10.1007/s11914-017-0375-y

12. Nuño C, Heili Frades Zimmermann SB, Alonso J et al (2001) Melorreostosis: presentación de un caso y revisión de la literatura. Rev Española Enfermedades Metabólicas Oseas 10(2):50-55 ISSN-e 1132-8460

13. Whyte M. "Osteonecrosis, osteosclerosis/hyperostosis, and other disorders of bone". Goldman's Cecil Medicine: Twenty Fourth Edition. Elsevier Inc; 2011. p. 1605-10. https://www.elsevier.com/books/goldmans-cecil-medicine/ 9781437716047

14. Teoh CC, Teoh SRZ, Chieng DCH et al (2019) Polyostotic melorheostosis: approach to clinical evaluation and management. Open J Orthop 09:137144. https://doi.org/10.4236/ojo.2019.97014

15. Hollick RJ, Black A, Reid D (2010) Melorheostosis and its treatment with intravenous zoledronic acid. BMJ Case Rep 2010:bcr0420091757. https://doi. org/10.1136/bcr.04.2009.1757

16. Dähnert W (2017) Musculoskeletal system: differential diagnosis of musculoskeletal disorders. In: Radiology review manual, 8th edn. Wolters Kluwer, Philadelphia, pp 46-60

\section{Publisher's Note}

Springer Nature remains neutral with regard to jurisdictional claims in published maps and institutional affiliations.

\section{Submit your manuscript to a SpringerOpen ${ }^{\bullet}$ journal and benefit from:}

- Convenient online submission

- Rigorous peer review

- Open access: articles freely available online

High visibility within the field

- Retaining the copyright to your article

Submit your next manuscript at $>$ springeropen.com 